\title{
Evolution of a geometric constant along the Ricci flow
}

Guangyue Huang* and Zhi Li

\begin{tabular}{l}
\hline${ }^{\text {CCorrespondence: }}$ \\
hgy@henannu.edu.cn \\
Henan Engineering Laboratory for \\
Big Data Statistical Analysis and \\
Optimal Control, College of \\
Mathematics and Information \\
Science, Henan Normal University, \\
Xinxiang, Henan 453007, People's \\
Republic of China
\end{tabular}

${ }^{*}$ Correspondence:

hgy@henannu.edu.cn

Big Data Statistical Analysis and

Optimal Control, College of

Mathematics and Information

Republic of China

\begin{abstract}
In this paper, we establish the first variation formula of the lowest constant $\lambda_{a}^{b}(g)$ along the Ricci flow and the normalized Ricci flow, such that to the following nonlinear equation there exist positive solutions:

$$
-\Delta u+a u \log u+b R u=\lambda_{a}^{b} u
$$

with $\int_{M} u^{2} d v=1$, where $a$ is a real constant. In particular, the results proved in this paper generalize partial results in Cao (Proc. Am. Math. Soc. 136:4075-4078, 2008) and Li (Math. Ann. 338:927-946, 2007).
\end{abstract}

MSC: 58C40; 53C44

Keywords: Ricci flow; normalized Ricci flow; conjugate heat equation

\section{Introduction}

Let $(M, g)$ be an $n$-dimensional compact Riemannian manifold. In [3], Perelman introduced the functional

$$
\mathcal{F}(g, f)=\int_{M}\left(|\nabla f|^{2}+R\right) e^{-f} d v
$$

and proved that the $\mathcal{F}$-functional is nondecreasing under the Ricci flow coupled to a backward heat-type equation

$$
\left\{\begin{array}{l}
\frac{\partial}{\partial t} g_{i j}=-2 R_{i j}, \\
f_{t}=-\Delta f+|\nabla f|^{2}-R,
\end{array}\right.
$$

where $R$ is the scalar curvature depending on the metric $g$. More precisely, they proved that under the system (1.2),

$$
\frac{d}{d t} \mathcal{F}=2 \int_{M}\left|R_{i j}+f_{i j}\right|^{2} e^{-f} d v \geq 0
$$

If we define

$$
\lambda(g)=\inf _{f} \mathcal{F}(g, f),
$$

(c) 2016 Huang and Li. This article is distributed under the terms of the Creative Commons Attribution 4.0 International License (http://creativecommons.org/licenses/by/4.0/), which permits unrestricted use, distribution, and reproduction in any medium, provided you give appropriate credit to the original author(s) and the source, provide a link to the Creative Commons license, and indicate if changes were made. 
where the infimum is taken over all smooth functions $f$ which satisfy

$$
\int_{M} e^{-f} d v=1
$$

then the nondecreasing of the $\mathcal{F}$-functional implies the nondecreasing of $\lambda(g)$. In particular, $\lambda(g)$ defined in (1.4) is the lowest eigenvalue of the operator

$$
-4 \Delta+R
$$

In [4], Cao considered the eigenvalues of the operator $-\Delta+\frac{R}{2}$ on manifolds with nonnegative curvature operator and showed that the eigenvalues are nondecreasing along the Ricci flow. Using the same technique, $\mathrm{Li}$ [2] also obtained the same monotonicity of the first eigenvalue of the operator $-\Delta+\frac{R}{2}$ by removing the assumption on a nonnegative curvature operator.

Later, Cao [1] proved the first eigenvalues of the operator $-\Delta+b R$ with the constant $b \geq 1 / 4$ are nondecreasing along the Ricci flow. That is, they assume $u=u(x, t)$ is the corresponding positive eigenfunction of $\lambda(t)$ :

$$
(-\Delta+b R) u=\lambda^{b} u
$$

with $\int_{M} u^{2} d v=1$, then

$$
\frac{d}{d t} \lambda^{b}=\frac{1}{2} \int_{M}\left|R_{i j}+f_{i j}\right|^{2} e^{-f} d v+\left(2 b-\frac{1}{2}\right) \int_{M}\left|R_{i j}\right|^{2} e^{-f} d v \geq 0
$$

by letting $f=-2 \log u$. Multiplying both sides of (1.7) with $u$ and integrating on $M$, we see that the first eigenvalue given in (1.7) satisfies

$$
\lambda(t)=\inf \tilde{\mathcal{F}}^{b}(g, u),
$$

where

$$
\tilde{\mathcal{F}}^{b}(g, u)=\int_{M}\left(|\nabla u|^{2}+b R u^{2}\right) d v
$$

In particular,

$$
\tilde{\mathcal{F}}^{b}(g, u)=\frac{1}{4} \mathcal{F}^{4 b}(g, f),
$$

where

$$
\mathcal{F}^{c}(g, f)=\int_{M}\left(|\nabla f|^{2}+c R\right) e^{-f} d v
$$

if we let $f=-2 \log u$. It is easy to see from (1.11) that the nondecreasing of the $\tilde{\mathcal{F}}^{b}$-functional is equivalent to the nondecreasing of $\lambda(t)$. 
In this paper, we consider the monotonicity along the Ricci flow of lowest constant $\lambda_{a}^{b}(g)$ such that to the following nonlinear equation there exist positive solutions:

$$
-\Delta u+a u \log u+b R u=\lambda_{a}^{b} u
$$

with

$$
\int_{M} u^{2} d v=1
$$

where $a$ is a real constant. In particular, (1.7) can be seen a special case of (1.12) when $a=0$. For the lowest constant $\lambda_{a}^{b}(g)$ such that to the nonlinear equation (1.12) there exist positive solutions, we prove the following.

Theorem 1.1 Let $g(t), t \in[0, T)$ be a solution to the Ricci flow

$$
\frac{\partial}{\partial t} g_{i j}=-2 R_{i j}
$$

on a compact Riemannian manifold $M$. Then for $b \geq \frac{1}{4}$, the lowest constant $\lambda_{a}^{b}(g)$ such that to the nonlinear equation (1.12) with (1.13) there exist positive solutions satisfies

$$
\begin{aligned}
\frac{d}{d t}\left(\lambda_{a}^{b}(t)+\frac{n a^{2}}{8} t\right) & =\frac{1}{2} \int_{M}\left|R_{i j}+f_{i j}+\frac{a}{2} g_{i j}\right|^{2} e^{-f} d v+\left(2 b-\frac{1}{2}\right) \int_{M}\left|R_{i j}\right|^{2} e^{-f} d v \\
& \geq 0,
\end{aligned}
$$

where $f=-2 \log u$.

For the normalized Ricci flow, we can obtain the following.

Theorem 1.2 Let $g(t), t \in[0, T)$ be a solution to the normalized Ricci flow

$$
\frac{\partial}{\partial t} g_{i j}=-2\left(R_{i j}-\frac{r}{n} g_{i j}\right)
$$

on a compact Riemannian manifold $M$, where $r=\left(\int_{M} R d v\right) /\left(\int_{M} d v\right)$ is the average scalar curvature. Then the lowest constant $\lambda_{a}^{b}(g)$ such that to the nonlinear equation (1.12) with (1.13) there exist positive solutions satisfies

$$
\begin{aligned}
\frac{d}{d t}\left(\lambda_{a}^{b}+\frac{n a^{2}}{8} t\right)+\frac{2 r}{n} \lambda^{b}= & \frac{1}{2} \int_{M}\left|R_{i j}+f_{i j}+\frac{a}{2} g_{i j}\right|^{2} e^{-f} d v \\
& +\left(2 b-\frac{1}{2}\right) \int_{M}\left|R_{i j}\right|^{2} e^{-f} d v
\end{aligned}
$$

where $f=-2 \log u$ and $\lambda^{b}$ is the lowest eigenvalue of (1.7).

In particular, when $n=2$, we have $R_{i j}=\frac{R}{2} g_{i j}$ and the normalized Ricci flow (1.16) becomes $\frac{\partial}{\partial t} g_{i j}=-(R-r) g_{i j}$. Hence, $\frac{d}{d t} r=0$, which implies that $r$ is a constant (or see p.455 in [5] for an alternative proof). Then from the estimate (1.17), we obtain the following. 
Theorem 1.3 Let $g(t), t \in[0, T)$ be a solution to the normalized Ricci flow (1.16) on a compact surface $M^{2}$. Then for $b \geq \frac{1}{4}$, the lowest constant $\lambda_{a}^{b}(g)$ such that to the nonlinear equation (1.12) with (1.13) there exist positive solutions satisfies

$$
\begin{aligned}
\frac{d}{d t}\left(\lambda_{a}^{b}+\frac{a^{2}}{4} t+r \int_{0}^{t} \lambda^{b}(s) d s\right)= & \frac{1}{2} \int_{M}\left|R_{i j}+f_{i j}+\frac{a}{2} g_{i j}\right|^{2} e^{-f} d v \\
& +\left(2 b-\frac{1}{2}\right) \int_{M}\left|R_{i j}\right|^{2} e^{-f} d v \\
\geq & 0,
\end{aligned}
$$

where $f=-2 \log u$ and $\lambda^{b}$ is the lowest eigenvalue of (1.7).

Remark 1.1 In particular, when $a=0$, our estimate (1.15) reduces to Theorem 1.5 of Cao in [1] and the estimate (1.18) reduces to the Corollary 2.4 of Cao in [1], respectively.

On the other hand, under the transformation $f=-2 \log u=-\log v$ with $u^{2}=v$, equation (1.2) becomes

$$
\left\{\begin{array}{l}
\frac{\partial}{\partial t} g_{i j}=-2 R_{i j} \\
v_{t}=-\Delta v+R v
\end{array}\right.
$$

In particular, the second equation in (1.19) is exactly the conjugate heat equation introduced by Perelman. In [6], Cao and Zhang obtained differential Harnack inequalities for positive solutions of the nonlinear parabolic equation of the type $v_{t}=\Delta v-v \log v+R v$. Extending the second equation in (1.19) to the following nonlinear version:

$$
v_{t}=-\Delta v+a v \log v+S v
$$

Guo and Ishida [7, 8] studied Harnack inequalities for positive solutions of equation (1.20) on a compact Riemannian manifold with a family of $g(t)$ evolving by a geometric flow $\frac{\partial}{\partial t} g_{i j}=-2 S_{i j}$, where $S_{i j}$ is a family of smooth symmetric two-tensor and $S=g^{i j} S_{i j}$. Clearly, there is a one-to-one relation for the following two equations:

$$
\frac{\partial}{\partial t} v=-\Delta v+a v \log v+R v \quad \Longleftrightarrow \quad \frac{\partial}{\partial t} f=-\Delta f+|\nabla f|^{2}+a f-R
$$

under $f=-\log v$. Therefore, a natural problem is to consider the monotonicity of

$$
\overline{\mathcal{F}}_{d}^{c}(g, f)=\int_{M}\left[|\nabla f|^{2}+c R+d(f+1)\right] e^{-f} d v
$$

under the Ricci flow coupled to a nonlinear backward heat-type equation

$$
\left\{\begin{array}{l}
\frac{d}{d t} g_{i j}=-2 R_{i j}, \\
f_{t}=-\Delta f+|\nabla f|^{2}+a f-R,
\end{array}\right.
$$

where $c, d$ are two real constants. 
For the functional $\overline{\mathcal{F}}_{d}^{c}(g, f)$, we derive the following monotonicity formula.

Theorem 1.4 Let $g(t), t \in[0, T)$ be a solution to the Ricciflow (1.14) on a compact Riemannian manifold $M$. Then all functionals $\overline{\mathcal{F}}_{d}^{c}(g, f)$ defined by (1.22) under the system (1.23) satisfy

$$
\begin{aligned}
\frac{d}{d t} \overline{\mathcal{F}}_{\frac{n a k}{8}}^{k}(g, f)= & 2 \int_{M}\left|R_{i j}+f_{i j}-\frac{a}{4} f g_{i j}\right|^{2} e^{-f} d v+2(k-1) \int_{M}\left|R_{i j}-\frac{a}{4} f g_{i j}\right|^{2} e^{-f} d v \\
& +\frac{n a}{8} k \mathcal{F}^{1}(g, f)+a \mathcal{F}^{0}(g, f) .
\end{aligned}
$$

In particular, if $R(t) \geq 0$ for all $t$ and $a \geq 0, k \geq 1$, then $\frac{d}{d t} \overline{\mathcal{F}}_{\frac{n a k}{8}}^{k}(g, f) \geq 0$.

Remark 1.2 Choosing $a=0$ in (1.24), we obtain Theorem 4.2 of $\mathrm{Li}$ in [2].

\section{Proof of Theorems 1.1 and 1.2}

Proof of Theorems 1.1 Let $u$ be a positive solution to the following nonlinear elliptic equation:

$$
-\Delta u+a u \log u+b R u=\lambda_{a}^{b} u
$$

Multiplying both sides of (2.1) with $u$ and integrating on $M$, we have

$$
\lambda_{a}^{b}=\int_{M}\left(|\nabla u|^{2}+a u^{2} \log u+b R u^{2}\right) d v .
$$

If the metric $g(t)$ evolves by (1.14), we have $\frac{\partial}{\partial t} d v=-R d v$. It follows from (2.2) that

$$
\begin{aligned}
\frac{d}{d t} \lambda_{a}^{b}= & \int_{M}\left(2 R_{i j} u^{i} u^{j}+2\left(u_{t}\right)^{i} u_{i}+2 a u u_{t} \log u+a u u_{t}+b R_{t} u^{2}+2 b R u u_{t}\right) d v \\
& -\int_{M}\left(|\nabla u|^{2}+a u^{2} \log u+b R u^{2}\right) R d v
\end{aligned}
$$

Applying

$$
2 \int_{M} R_{i j} u^{i} u^{j} d v=\int_{M}\left(-R_{, i} u^{i} u-2 R_{i j} u^{i j} u\right) d v
$$

and

$$
-\int_{M}|\nabla u|^{2} R d v=\int_{M}\left(R \Delta u+R_{, i} u^{i}\right) u d v
$$

into (2.3) yields

$$
\begin{aligned}
\frac{d}{d t} \lambda_{a}^{b}= & \int_{M}\left[-2 R_{i j} u^{i j} u+b R_{t} u^{2}+a u u_{t}\right. \\
& \left.+2 u_{t}(-\Delta u+a u \log u+b R u)-R u(-\Delta u+a u \log u+b R u)\right] d v
\end{aligned}
$$




$$
\begin{aligned}
& =\int_{M}\left[-2 R_{i j} u^{i j} u+b R_{t} u^{2}+\frac{a}{2}\left(u^{2}\right)_{t}\right] d v+\lambda\left(\int_{M} u^{2} d v\right)_{t} \\
& =\int_{M}\left[-2 R_{i j} u^{i j} u+b R_{t} u^{2}+\frac{a}{2} R u^{2}\right] d v
\end{aligned}
$$

where the last equality used

$$
\int_{M}\left[\left(u^{2}\right)_{t}-R u^{2}\right] d v=0
$$

from (1.13). Noticing $R_{t}=\Delta R+2\left|R_{i j}\right|^{2}$ for the Ricci flow, hence from (2.6) we have

$$
\begin{aligned}
\frac{d}{d t} \lambda_{a}^{b} & =\int_{M}\left[-2 R_{i j} u^{i j} u+b u^{2}\left(\Delta R+2\left|R_{i j}\right|^{2}\right)+\frac{a}{2} R u^{2}\right] d v \\
& =\int_{M}\left[-2 R_{i j} u^{i j} u+b R \Delta\left(u^{2}\right)+2 b\left|R_{i j}\right|^{2} u^{2}+\frac{a}{2} R u^{2}\right] d v .
\end{aligned}
$$

Taking a transformation $f=-2 \log u$, which is equivalent to $u^{2}=e^{-f}$, then

$$
u^{i j}=\left(-\frac{1}{2} f^{i j}+\frac{1}{4} f^{i} f^{j}\right) e^{-\frac{f}{2}} .
$$

Thus, (2.8) can be written as

$$
\frac{d}{d t} \lambda_{a}^{b}=\int_{M}\left[R_{i j} f^{i j}-\frac{1}{2} R_{i j} f^{i} f^{j}-b R \Delta f+b R|\nabla f|^{2}+2 b\left|R_{i j}\right|^{2}+\frac{a}{2} R\right] e^{-f} d v .
$$

Using the second Bianchi identity $R_{, i}=2 R_{i j}{ }^{j}$ again, we have

$$
\begin{aligned}
-b \int_{M} R \Delta f e^{-f} d v & =\int_{M}\left(b R_{, i} f^{i}-b R|\nabla f|^{2}\right) e^{-f} d v \\
& =\int_{M}\left(-2 b R_{i j} f^{i j}+2 b R_{i j} f^{i} f^{j}-b R|\nabla f|^{2}\right) e^{-f} d v .
\end{aligned}
$$

Therefore, inserting (2.11) into (2.10) yields

$$
\begin{aligned}
\frac{d}{d t} \lambda_{a}^{b}= & (1-2 b) \int_{M} R_{i j} f^{i j} e^{-f} d v+\left(2 b-\frac{1}{2}\right) \int_{M} R_{i j} f^{i} f^{j} e^{-f} d v \\
& +2 b \int_{M}\left|R_{i j}\right|^{2} e^{-f} d v+\frac{a}{2} \int_{M} R e^{-f} d v .
\end{aligned}
$$

Integrating by parts again, one has

$$
\int_{M} R_{i j} f^{i j} e^{-f} d v=\int_{M} R_{i j} f^{i} f^{j} e^{-f} d v-\frac{1}{2} \int_{M} R \Delta e^{-f} d v
$$

and

$$
\begin{aligned}
& \int_{M} R_{i j} f^{i j} e^{-f} d v+\int_{M}\left|f_{i j}\right|^{2} e^{-f} d v \\
& \quad=\frac{1}{2} \int_{M} \Delta|\nabla f|^{2} e^{-f} d v-\int_{M}(\Delta f)_{i} f^{i} e^{-f} d v-\frac{1}{2} \int_{M} R \Delta e^{-f} d v
\end{aligned}
$$




$$
\begin{aligned}
& =-\int_{M}\left[\Delta f-\frac{1}{2}|\nabla f|^{2}+\frac{1}{2} R\right] \Delta e^{-f} d v \\
& =\left(2 b-\frac{1}{2}\right) \int_{M} R \Delta e^{-f} d v-a \int_{M}|\nabla f|^{2} e^{-f} d v,
\end{aligned}
$$

where the last equality in (2.14) was used with

$$
2 \lambda_{a}^{b}=\Delta f-\frac{1}{2}|\nabla f|^{2}-a f+2 b R
$$

By virtue of (2.14), subtracting (2.13), we obtain

$$
\int_{M}\left|f_{i j}\right|^{2} e^{-f} d v=2 b \int_{M} R \Delta e^{-f} d \nu-\int_{M} R_{i j} f^{i} f^{j} e^{-f} d v-a \int_{M}|\nabla f|^{2} e^{-f} d \nu
$$

It follows from (2.13) and (2.14) that

$$
\begin{aligned}
\frac{d}{d t} \lambda_{a}^{b}= & (1-2 b) \int_{M} R_{i j} f^{i j} e^{-f} d v+\left(2 b-\frac{1}{2}\right) \int_{M} R_{i j} f^{i} f^{j} e^{-f} d v \\
& +2 b \int_{M}\left|R_{i j}\right|^{2} e^{-f} d v+\frac{a}{2} \int_{M} R e^{-f} d v \\
= & \int_{M} R_{i j} f^{i j} e^{-f} d v-\frac{1}{2} \int_{M} R_{i j} f^{i} f^{j} e^{-f} d v \\
& +2 b \int_{M}\left|R_{i j}\right|^{2} e^{-f} d v+\frac{a}{2} \int_{M} R e^{-f} d v+b \int_{M} R \Delta e^{-f} d v \\
= & \int_{M} R_{i j} f^{i j} e^{-f} d v+2 b \int_{M}\left|R_{i j}\right|^{2} e^{-f} d v+\frac{a}{2} \int_{M} R e^{-f} d v \\
& +\frac{1}{2} \int_{M}\left|f_{i j}\right|^{2} e^{-f} d v+\frac{a}{2} \int_{M}(\Delta f) e^{-f} d v \\
= & \frac{1}{2} \int_{M}\left|R_{i j}+f_{i j}+\frac{a}{2} g_{i j}\right|^{2} e^{-f} d v+\left(2 b-\frac{1}{2}\right) \int_{M}\left|R_{i j}\right|^{2} e^{-f} d v \\
& -\frac{n a^{2}}{8},
\end{aligned}
$$

and the desired estimate (1.15) is achieved.

Proof of Theorem 1.2 If the metric $g(t)$ evolves by (1.16), we have $\frac{\partial}{\partial t} d v=-(R-r) d v$. It follows from (2.2) that

$$
\begin{aligned}
\frac{d}{d t} \lambda_{a}^{b}= & \int_{M}\left(2 R_{i j} u^{i} u^{j}-\frac{2 r}{n}|\nabla u|^{2}+2\left(u_{t}\right)^{i} u_{i}+2 a u u_{t} \log u+a u u_{t}+b R_{t} u^{2}\right. \\
& \left.+2 b R u u_{t}\right) d v-\int_{M}\left(|\nabla u|^{2}+a u^{2} \log u+b R u^{2}\right)(R-r) d v
\end{aligned}
$$

Applying (2.4) and

$$
-\int_{M}|\nabla u|^{2}(R-r) d v=\int_{M}\left[(R-r) \Delta u+R_{, i} u^{i}\right] u d v
$$


to $(2.18)$ yields

$$
\begin{aligned}
\frac{d}{d t} \lambda_{a}^{b}= & \int_{M}\left[-2 R_{i j} u^{i j} u-\frac{2 r}{n}|\nabla u|^{2}+b R_{t} u^{2}+a u u_{t}\right. \\
& \left.+2 u_{t}(-\Delta u+a u \log u+b R u)-(R-r) u(-\Delta u+a u \log u+b R u)\right] d v \\
= & \int_{M}\left[-2 R_{i j} u^{i j} u-\frac{2 r}{n}|\nabla u|^{2}+b R_{t} u^{2}+\frac{a}{2}\left(u^{2}\right)_{t}\right] d v+\lambda\left(\int_{M} u^{2} d v\right)_{t} \\
= & \int_{M}\left[-2 R_{i j} u^{i j} u-\frac{2 r}{n}|\nabla u|^{2}+b R_{t} u^{2}+\frac{a}{2} R u^{2}\right] d v .
\end{aligned}
$$

Noticing $R_{t}=\Delta R+2\left|R_{i j}\right|^{2}-\frac{2 r}{n} R$ for the normalized Ricci flow, we obtain from (2.20)

$$
\begin{aligned}
\frac{d}{d t} \lambda_{a}^{b}= & \int_{M}\left[-2 R_{i j} u^{i j} u-\frac{2 r}{n}|\nabla u|^{2}+b R_{t} u^{2}+\frac{a}{2} R u^{2}\right] d v \\
= & \int_{M}\left[-2 R_{i j} u^{i j} u-\frac{2 r}{n}|\nabla u|^{2}+b u^{2}\left(\Delta R+2\left|R_{i j}\right|^{2}-\frac{2 r}{n} R\right)+\frac{a}{2} R u^{2}\right] d v \\
= & \int_{M}\left[-2 R_{i j} u^{i j} u+b R \Delta\left(u^{2}\right)+2 b\left|R_{i j}\right|^{2} u^{2}+\frac{a}{2} R u^{2}\right] d v \\
& -\frac{2 r}{n} \int_{M}\left(|\nabla u|^{2}+b R u^{2}\right) d v
\end{aligned}
$$

Using (2.9), then (2.21) can be written as

$$
\begin{aligned}
\frac{d}{d t} \lambda_{a}^{b}= & \int_{M}\left[R_{i j} f^{i j}-\frac{1}{2} R_{i j} f^{i} f^{j}-b R \Delta f+b R|\nabla f|^{2}+2 b\left|R_{i j}\right|^{2}+\frac{a}{2} R\right] e^{-f} d v \\
& -\frac{2 r}{n} \int_{M}\left(\frac{1}{4}|\nabla f|^{2}+b R\right) e^{-f} d v
\end{aligned}
$$

By virtue of a similar computation, we can obtain

$$
\begin{aligned}
\frac{d}{d t} \lambda_{a}^{b}= & \frac{1}{2} \int_{M}\left|R_{i j}+f_{i j}+\frac{a}{2} g_{i j}\right|^{2} e^{-f} d \nu+\left(2 b-\frac{1}{2}\right) \int_{M}\left|R_{i j}\right|^{2} e^{-f} d \nu \\
& -\frac{n a^{2}}{8}-\frac{2 r}{n} \int_{M}\left(\frac{1}{4} \Delta f+b R\right) e^{-f} d v,
\end{aligned}
$$

which gives

$$
\begin{aligned}
\frac{d}{d t}\left(\lambda_{a}^{b}+\frac{n a^{2}}{8} t\right)+\frac{2 r}{n} \lambda^{b}= & \frac{1}{2} \int_{M}\left|R_{i j}+f_{i j}+\frac{a}{2} g_{i j}\right|^{2} e^{-f} d \nu \\
& +\left(2 b-\frac{1}{2}\right) \int_{M}\left|R_{i j}\right|^{2} e^{-f} d \nu
\end{aligned}
$$

Then the desired estimate (1.17) is attained. 


\section{Proof of Theorem 1.4}

Under the following coupled system (1.23), by a direct computation, we have the following:

$$
\begin{aligned}
\frac{\partial}{\partial t}\left(e^{-f} d v\right) & =-\left(f_{t}+R\right) e^{-f} d v=\left[\Delta f-|\nabla f|^{2}-a f\right] e^{-f} d v \\
& =-\left(\Delta e^{-f}\right) d v-a f e^{-f} d v \\
\frac{\partial}{\partial t}|\nabla f|^{2}= & 2 R^{i j} f_{i} f_{j}+2 f^{i}\left(f_{t}\right)_{i} \\
= & 2 R^{i j} f_{i} f_{j}+2 f^{i}\left(-\Delta f+|\nabla f|^{2}+a f-R\right)_{i} \\
= & 2 R^{i j} f_{i} f_{j}-2 f^{i}(\Delta f)_{i}+4 f^{i j} f_{i} f_{j}+2 a|\nabla f|^{2}-2 R_{i} f^{i}
\end{aligned}
$$

Thus, we have

$$
\begin{aligned}
\frac{d}{d t} \int_{M} e^{-f} d v & =-a \int_{M} f e^{-f} d v \\
\frac{d}{d t} \int_{M} R e^{-f} d v & =\int_{M}\left[\Delta R+2\left|R_{i j}\right|^{2}-a f R\right] e^{-f} d v-\int_{M} R\left(\Delta e^{-f}\right) d v \\
& =\int_{M}\left[2\left|R_{i j}\right|^{2}-a f R\right] e^{-f} d v \\
\frac{d}{d t} \int_{M} f e^{-f} d v & =\int_{M}(a f-R) e^{-f} d v-\int_{M} f\left(\Delta e^{-f}\right) d v-\int_{M} a f^{2} e^{-f} d v \\
& =\int_{M}\left[a f-a f^{2}-(R+\Delta f)\right] e^{-f} d v
\end{aligned}
$$

and

$$
\begin{aligned}
\frac{d}{d t} & \int_{M}|\nabla f|^{2} e^{-f} d v \\
= & \int_{M}\left[2 R^{i j} f_{i} f_{j}-2 f^{i}(\Delta f)_{i}+4 f^{i j} f_{i} f_{j}+2 a|\nabla f|^{2}-2 R_{i} f^{i}\right] e^{-f} d v \\
& -\int_{M}\left(\Delta e^{-f}\right)|\nabla f|^{2} d v-\int_{M} a f|\nabla f|^{2} e^{-f} d v \\
= & \int_{M}\left[-2 f_{i j}^{2}-4 f^{i}(\Delta f)_{i}+4 f^{i j} f_{i} f_{j}+2 a|\nabla f|^{2}-2 R_{i} f^{i}\right] e^{-f} d v \\
& -\int_{M} a f|\nabla f|^{2} e^{-f} d v .
\end{aligned}
$$

By virtue of the Bochner formula with respect to the $f$-Laplacian, we have

$$
\frac{1}{2} \Delta_{f}|\nabla u|^{2}=u_{i j}^{2}+u_{i}\left(\Delta_{f} u\right)_{i}+\left(R^{i j}+f^{i j}\right) u_{i} u_{j}, \quad \forall u,
$$

and hence

$$
\begin{aligned}
0 & =\int_{M}\left[f_{i j}^{2}+f_{i}\left(\Delta_{f} f\right)_{i}+\left(R^{i j}+f^{i j}\right) f_{i} f_{j}\right] e^{-f} d v \\
& =\int_{M}\left[f_{i j}^{2}+f_{i}(\Delta f)_{i}+R^{i j} f_{i} f_{j}-f^{i j} f_{i} f_{j}\right] e^{-f} d v .
\end{aligned}
$$


Therefore, (3.6) becomes

$$
\begin{aligned}
\frac{d}{d t} \int_{M}|\nabla f|^{2} e^{-f} d v & =\int_{M}\left[2 f_{i j}^{2}+4 R^{i j} f_{i} f_{j}+2 a|\nabla f|^{2}-2 R_{i} f^{i}\right] e^{-f} d v-\int_{M} a f|\nabla f|^{2} e^{-f} d v \\
& =\int_{M}\left[2 f_{i j}^{2}+4 R^{i j} f_{i j}+2 a|\nabla f|^{2}\right] e^{-f} d v-\int_{M} a(f+1)(\Delta f) e^{-f} d v
\end{aligned}
$$

Therefore, from (3.4) and (3.8), we obtain

$$
\begin{aligned}
\frac{d}{d t} \int_{M}\left(R+|\nabla f|^{2}\right) e^{-f} d v= & 2 \int_{M}\left|R_{i j}+f_{i j}-\frac{a}{4} f g_{i j}\right|^{2} e^{-f} d v \\
& -\frac{n a^{2}}{8} \int_{M} f^{2} e^{-f} d v+a \int_{M}|\nabla f|^{2} e^{-f} d v
\end{aligned}
$$

Noticing (3.5) tells us that

$$
-a \int_{M} f^{2} e^{-f} d v=\frac{d}{d t}\left(\int_{M}(f+1) e^{-f} d v\right)+\int_{M}(R+\Delta f) e^{-f} d \nu
$$

Thus, (3.9) can be written as

$$
\begin{aligned}
& \frac{d}{d t} \int_{M}\left[R+|\nabla f|^{2}+\frac{n a}{8}(f+1)\right] e^{-f} d v \\
& \quad=2 \int_{M}\left|R_{i j}+f_{i j}-\frac{a}{4} f g_{i j}\right|^{2} e^{-f} d v+\frac{n a}{8} \int_{M}\left(R+|\nabla f|^{2}\right) e^{-f} d v+a \int_{M}|\nabla f|^{2} e^{-f} d v
\end{aligned}
$$

Since (3.4) holds, we have

$$
\frac{d}{d t} \int_{M} R e^{-f} d \nu=2 \int_{M}\left|R_{i j}-\frac{a}{4} f g_{i j}\right|^{2} e^{-f} d \nu-\frac{n a^{2}}{8} \int_{M} f^{2} e^{-f} d \nu,
$$

which gives

$$
\begin{aligned}
\frac{d}{d t} \int_{M}\left[R+\frac{n a}{8}(f+1)\right] e^{-f} d v= & 2 \int_{M}\left|R_{i j}-\frac{a}{4} f g_{i j}\right|^{2} e^{-f} d v \\
& +\frac{n a}{8} \int_{M}\left(R+|\nabla f|^{2}\right) e^{-f} d v
\end{aligned}
$$

Therefore, we have

$$
\begin{aligned}
& \frac{d}{d t} \int_{M}\left\{|\nabla f|^{2}+k\left[R+\frac{n a}{8}(f+1)\right]\right\} e^{-f} d v \\
& =2 \int_{M}\left|R_{i j}+f_{i j}-\frac{a}{4} f g_{i j}\right|^{2} e^{-f} d v+2(k-1) \int_{M}\left|R_{i j}-\frac{a}{4} f g_{i j}\right|^{2} e^{-f} d v \\
& \quad+\frac{n a}{8} k \int_{M}\left(R+|\nabla f|^{2}\right) e^{-f} d v+a \int_{M}|\nabla f|^{2} e^{-f} d v
\end{aligned}
$$

and the desired estimate (1.24) is obtained. 


\section{Conclusions}

We establish the first variation formula of the lowest constant $\lambda_{a}^{b}(g)$ along the Ricci flow and the normalized Ricci flow, such that to the following nonlinear equation there exist positive solutions:

$$
-\Delta u+a u \log u+b R u=\lambda_{a}^{b} u
$$

with $\int_{M} u^{2} d v=1$, where $a$ is a real constant. Equation (4.1) can be seen as a nonlinear version of eigenvalue problem of the operator $-\Delta u+b R$. In particular, when $a=0$, our estimate (1.15) in Theorem 1.1 reduces to Theorem 1.5 of Cao in [1] and the estimate (1.18) in Theorem 1.3 reduces to the Corollary 2.4 of Cao in [1], respectively.

On the other hand, we obtained the first variation formula (1.24) of the functional

$$
\overline{\mathcal{F}}_{d}^{c}(g, f)=\int_{M}\left[|\nabla f|^{2}+c R+d(f+1)\right] e^{-f} d v
$$

under the Ricci flow coupled to a nonlinear backward heat-type equation

$$
\left\{\begin{array}{l}
\frac{d}{d t} g_{i j}=-2 R_{i j} \\
f_{t}=-\Delta f+|\nabla f|^{2}+a f-R
\end{array}\right.
$$

where $c, d$ are two real constants. In particular, when $a=0$ in (1.24), we obtain Theorem 4.2 of $\mathrm{Li}$ in [2].

\section{Competing interests}

The authors declare that they have no competing interests.

\section{Authors' contributions}

All authors contributed equally to the writing of this paper. All authors read and approved the final manuscript.

\section{Acknowledgements}

The research of the first author is supported by NSFC (No. 11371018, 11171091), Henan Provincial Core Teacher (No. 2013GGJS-057) and IRTSTHN (14IRTSTHN023). The research of the second author is partially supported by NSFC (No. 11401179) and Henan Provincial Education department (No. 14B110017).

Received: 14 November 2015 Accepted: 4 February 2016 Published online: 11 February 2016

\section{References}

1. Cao, X-D: First eigenvalues of geometric operators under the Ricci flow. Proc. Am. Math. Soc. 136, 4075-4078 (2008)

2. Li, J-F: Eigenvalues and energy functionals with monotonicity formulae under Ricci flow. Math. Ann. 338, 927-946 (2007)

3. Perelman, G: The entropy formula for the Ricci flow and its geometric applications. arXiv:math.DG/0211159

4. Cao, X-D: Eigenvalues of $\left(-\Delta+\frac{R}{2}\right)$ on manifolds with nonnegative curvature operator. Math. Ann. 337, $435-441$ (2007)

5. Cao, X-D, Hou, S, Ling, J: Estimate and monotonicity of the first eigenvalue under the Ricci flow. Math. Ann. 354, 451-463 (2012)

6. Cao, X-D, Zhang, Z: Differential Harnack estimates for parabolic equations. In: Complex and Differential Geometry. Springer Proc. Math., vol. 8, pp. 87-98 (2011)

7. Guo, H, Ishida, M: Harnack estimates for nonlinear backward heat equations in geometric flows. J. Funct. Anal. 267, 2638-2662 (2014)

8. Guo, H, Ishida, M: Harnack estimates for nonlinear heat equations with potentials in geometric flows. Manuscr. Math. 148, 471-484 (2015) 